\title{
MedienPädagogik
}

Zeitschrift für Theorie und Praxis der Medienbildung

Themenheft Nr. 41: Inklusiv-mediale Bildung in schulischen Kontexten. Eine interdisziplinäre Bestandsaufnahme

Herausgegeben von Christian Filk und Heike Schaumburg

\section{Anforderungsstrukturen inklusiv-medialen pädagogischen Handelns}

\author{
Estella Ferraro, Julia Gasterstädt und Johannes Wahl
}

\begin{abstract}
Zusammenfassung
Pädagogisches Handeln als Interaktion innerhalb eines Arbeitsbündnisses unterliegt diversen Anforderungsstrukturen, die jenseits dieser Interaktionssituationen verhandelt werden. Im Zuge struktureller Veränderungen auf gesellschaftlicher Ebene tauchen immer neue zusätzliche Anforderungen auf, die in das berufskulturelle Mandat integriert werden sollen. Der vorliegende Beitrag thematisiert aus einer primär systemtheoretischen Perspektive den Wandel von Anforderungsstrukturen, die durch die parallele Verhandlung der Megathemen Digitalisierung und Inklusion angestossen werden. Dabei liegt der Fokus sowohl auf den Veränderungen im Bildungssystem durch die strukturelle Kopplung mit anderen gesellschaftlichen Funktionssystemen als auch auf den Aushandlungsprozessen darin, was anhand der Digitalisierungs- und Inklusionsdiskurse aufgezeigt wird. Es wird herausgearbeitet, wie sich die Anforderungsstrukturen inklusiv-medialer Bildung gestalten und welche Rolle dabei die Erwartungen gesellschaftlicher Teilsysteme einnehmen. Der Beitrag zielt auf einen (selbst)kritischen Blick in Bezug auf die Verstrickung in die eigenen Systemlogiken und die damit verbundenen strukturellen Kopplungen.
\end{abstract}

Requirements for Inclusive Media Education

\begin{abstract}
Pedagogical action is subject to certain requirements within working alliances that are negotiated both within and outside of particular interactional situations. Due to structural transformations on a social level new requirements constantly evolve that need to be integrated into the professional mandate. From a predominant system theoretical perspective, this paper analyses the transformation of requirement structures for educational professionals focusing on those transformations caused by digitalisation and inclusion. This will be done by looking both at the changes taking place within educational systems through the structural coupling with other social functional systems as well as by looking at the processes of negotiation within those systems in the case of the digitalisation and inclusion discourses. It is shown how the requirement structures of inclusive-mediaeducation evolve and which role the expectations of different social subsystems take. The paper aims at giving a (self)critical perspective on the entanglement into own ways of system logic (Systemlogik) and the related structural couplings.
\end{abstract}




\section{Einleitung}

Pädagogisches Handeln unterliegt komplexen Anforderungsstrukturen, die sich auf verschiedenen Ebenen analysieren lassen. In diese sind Spannungsfelder eingelagert, die insbesondere im Zuge von strukturellen Veränderungen aufscheinen. In diesem Kontext verändern sich nicht nur bestehende Spannungsfelder, sondern es entstehen auch neue. Ein Zugang zur Beschreibung dieser Spannungsfelder eröffnet sich durch eine systemtheoretische Perspektive. Betrachtet man das Bildungssystem hinsichtlich seiner Leistung für andere gesellschaftliche Teilsysteme, so wird die Humanontogenese als integrativer Beitrag deutlich (Wahl 2017). In diesem Sinn befähigt dieses Funktionssystem die Individuen bspw. zur Teilhabe an kulturellen, politischen Aushandlungs- und ökonomischen Prozessen. Im Gegenzug stehen andere Funktionssysteme wie etwa das Wirtschaftssystem über finanzielle Ressourcen mit ihm in Verbindung.

Vor dem Hintergrund solcher und weiterer struktureller Kopplungen (Luhmann 1997) lassen sich die Auswirkungen politischer Entscheidungen auf Schwerpunktsetzungen innerhalb des Bildungssystems erfassen. Ausgehend von strukturellen Veränderungen in postmodernen Gesellschaften (Bauman 2009; Castells 2017) wird dieses Funktionssystem und die in ihm tätigen Professionellen mit immer neuen Anforderungen konfrontiert. Sie lassen sich aufgrund ihrer Herkunft aus anderen Funktionssystemen als Anforderungsstrukturen verstehen, unter deren Wirksamkeit pädagogisch Professionelle ihrer beruflichen Tätigkeit nachgehen. Dazu zählt bspw. das Phänomen der zunehmenden Entstandardisierung von Normal(erwerbs-)biografien (Hof 2009). Darüber hinaus lassen sich auch politische Akzentuierungen am Beispiel des lebenslangen Lernens (Wahl 2017), der inklusiven Gestaltung des Bildungssystems (Gasterstädt 2019) sowie an Phänomenen der Digitalgesellschaft (Damberger 2018) als Anforderungen begreifen, die einen Einfluss auf pädagogisches Handeln ausüben.

Der Beitrag thematisiert die veränderten Anforderungsstrukturen an pädagogisches Handeln in Hinblick auf die Megathemen Inklusion und Digitalisierung und beschreibt, wie sich Prozesse des Wandels professionellen Handelns im Hinblick auf diese Anforderungsstrukturen beschreiben lassen. Bearbeitet wird diese Fragestellung, indem zunächst eine professionstheoretische Perspektive auf die sich wandelnden Anforderungen an pädagogisch Tätige eröffnet wird. Anschliessend werden die veränderten Anforderungsstrukturen, die mit den beiden Themen einhergehen, skizziert und in Bezug zueinander gesetzt, um diese sodann hinsichtlich der Forderung nach Reflexion zur Bearbeitung der Spannungsfelder pädagogischen Handelns diskutieren zu können. 


\section{Konstitution von Anforderungsstrukturen}

Aus der Vielfalt an professionstheoretischen Sichtweisen auf pädagogisches Handeln lassen sich systemtheoretisch relevante Aspekte, wie die Frage nach der Funktion von Berufskulturen in gesellschaftlichen Teilsystemen, durch den Bezug auf klassische Arbeiten von Hughes (1993) und Stichweh (1996) rahmen. Durch diesen Blick auf die pädagogisch Tätigen innerhalb des Bildungssystems wird vor allem eines ihrer strukturellen Merkmale deutlich. Es zeichnet sich «durch die Aushandlung eines gesellschaftlichen Mandats (Auftrag) und einer gesellschaftlich ratifizierten Lizenz (Erlaubnis) aus» (Nittel 2011, 42). Dies macht deutlich, dass die Zuständigkeiten pädagogischen Handelns als gesellschaftlich verhandeltes Konstrukt begriffen werden können. So wird hinsichtlich des Mandats, dessen Bedeutung aus der exklusiven personenbezogenen Dienstleistung für das Gemeinwesen ableitbar ist (Nittel und Schütz 2013), erkennbar, dass dessen Ausrichtung unmittelbar mit den Erwartungen bzw. Zuschreibungen verschiedener Akteursgruppen verknüpft ist.

Gerade in komplexen Gesellschaften zeigt sich, dass die Vielfalt an institutionalisierten Erwartungshaltungen an die jeweilige Berufskultur zu Unschärfen und Ambivalenzen in den Konturen des Mandats führen kann. Dieses Phänomen resultiert nicht nur in Diskussionen innerhalb der jeweiligen Berufskultur zu konkreten Inhalten des eigenen Arbeitsauftrags, sondern kann auch Anstoss zur Veränderung dieser sozialen Gruppierung sein. So gerät der Wandel von Berufskulturen in Auseinandersetzung mit den jeweiligen Umweltbedingungen (Nittel und Seltrecht 2008) in den Blick. Ursprünglich mit dem Ziel verbunden, berufskulturelle Entwicklungsprozesse hin zum Status als Profession zu rahmen, zeigen empirische Beobachtungen eher problematische Konstellationen auf, die genau diesen Konstitutionsprozess prägen. Dabei wird deutlich, dass der Wandel sowohl semi- und protoprofessionelle Berufskulturen als auch klassische Professionen betrifft (Geissler 2013).

Um die Wandlungsprozesse, die sich unter anderem auf die Konstitution des Mandats auswirken, in den jeweiligen Berufskulturen analytisch zu erfassen, lässt sich das Konzept der sozialen Welt von Nittel (2011) heranziehen. In Anknüpfung an Strauss (1993) benutzt er dieses, um die Vielfalt der Berufskulturen in pädagogischen Arbeitskontexten analytisch fassbar zu machen:

«Die soziale Welt pädagogisch Tätiger kann mit dem Arsenal folgender analytischer Begriffe untersucht und verglichen werden: berufliche Sinnquellen, Kernaktivitäten, Arbeitsbögen, biographische Basisdispositionen und berufliche Basispositionen, Lizenz und Mandat, Arenen (Innen- und Aussenarenen), pädagogische Technologien.» (Nittel 2011, 52)

Durch den Bezug zu Innen- und Aussenarenen wird deutlich, dass die sozialen Welten als wandelbare soziale Einheiten verstanden werden, deren Struktur sowohl durch Aushandlungsprozesse innerhalb (Innenarenen) als auch jenseits der eigenen 
Berufskultur (Aussenarenen) veränderbar ist. Mit Blick auf die letztgenannte Möglichkeit zur Veränderung der Berufskultur schliesst sich unmittelbar die Frage an, mit welchen Akteursgruppen die Aushandlungsprozesse vollzogen werden. Ein Hinweis darauf findet sich bspw. bei Tippelt:

«Pädagogisch professionelles Handeln basiert auf der Reflektion gesellschaftlicher Rahmenbedingungen, fokussiert pädagogische Ideen und integriert die verfügbaren Ergebnisse der empirischen Bildungsforschung.» (Tippelt 2018, 649)

Der kurze Auszug verdeutlicht die Verwobenheit des Bildungssystems mit anderen gesellschaftlichen Funktionssystemen. So verweist der Autor auf den spezifischen Bezug zu Rahmenbedingungen, die bspw. durch (bildungs-)politische Akzentsetzungen geformt werden, und verdeutlicht die strukturelle Kopplung insbesondere mit dem Wissenschaftssystem bzw. dessen Leistung.

Die Verbindungen zwischen (Erziehungs-)Wissenschaft, (Bildungs-)Politik und (pädagogischer) Praxis verweisen nicht nur auf den Prozess der Ausdifferenzierung von Funktionssystemen. Vielmehr lässt sich damit erläutern, inwiefern die Erwartungen spezifischer Akteursgruppen an professionelles pädagogisches Handeln zur Veränderung des Mandats beitragen können. Durch den Fokus auf derartige Anspruchshaltungen geraten die bereits oben erwähnten Anforderungsstrukturen in den Blick. Auch sie werden innerhalb wie ausserhalb spezifischer Funktionssysteme eigenlogisch verhandelt und unterliegen kontinuierlichen Veränderungen. Der Gegenstand dieser Veränderungen ist wiederum von verschiedenen Megathemen abhängig, die mit dem Zuständigkeitsbereich des pädagogischen Handelns verknüpft werden. Die daraus ableitbaren Implikationen für pädagogisch Tätige werden nachfolgend anhand der beiden Themenfelder Digitalisierung und Inklusion diskutiert und hinsichtlich ihrer Bedeutung für inklusiv-mediales pädagogisches Handeln analysiert.

\section{Digitalisierung? Digitalisierung!}

Es lässt sich aktuell nicht bestreiten, dass den Medien und Informationstechnologien ein hoher Stellenwert nicht nur in unterschiedlichen Diskursen, sondern auch im alltäglichen Leben zukommt (Bettinger 2018). Dies lässt sich zum Beispiel an der Diskussion zum DigitalPakt Schule (BMBF 2019) zeigen, die sich sowohl auf politischer, schulischer und wissenschaftlicher Ebene als auch in öffentlichen Berichterstattungen wiederfinden lässt. Dander zeigt dabei auf, dass häufig zwischen Digitalisierung und der Rede von Digitalisierung unterschieden werden kann (Dander 2020). Es geht also zum einen um den Diskurs und zum anderen um den Prozess der Digitalisierung an sich. Dies scheint besonders bei den Themen der Digitalisierung als auch der Inklusion in Anbetracht der aktuellen Beliebtheit dieser Begriffe wichtig. 
Auf der Ebene der Medienpädagogik ergibt sich aus dem Gedanken die folgende Ambivalenz: Zum einen spielt der technische Fortschritt eine zentrale Rolle und beeinflusst die Anforderungsstrukturen für pädagogisch Tätige. Zum anderen ist auch der Diskurs um den Fortschritt und die damit einhergehenden Anforderungsstrukturen im ständigen Wandel. Aus ihm können relevante Aspekte und Anforderungsbedarfe resultieren. Im Folgenden wird mit Fokus auf den medienpädagogischen Diskurs aufgezeigt, inwiefern Anforderungsstrukturen an pädagogisch Tätige aus den aktuellen Bedingungen heraus entstehen und inwiefern diese stellenweise an sich schon paradox erscheinen können. Dafür wird zunächst der theoretische Bezugsrahmen zur Medienbildung deutlich gemacht.

«Medien bestimmen wesentlich die Strukturen von Weltsichten, sowohl auf kultureller Ebene wie auch auf individueller Ebene» (Jörissen 2013). Marotzki und Jörissen machen in ihren Ansätzen zur strukturalen Medienbildung (2009) deutlich, dass Weltsichten sich immer schon medial konstituiert haben. Dies sei den Autoren nach nicht nur auf kultureller, sondern auch auf individueller Ebene der Fall. In Abhängigkeit der verschiedenen kulturellen Praktiken entstehen so unterschiedliche Möglichkeiten von Artikulation (Marotzki und Jörissen 2008). Das menschliche Sein in der Welt ist demnach immer medial geprägt. In diesem Sinne setzen die Autoren den Bildungsbegriff mit dem der Medien in Verbindung, da sich Bildungsprozesse in einer von «Medien durchzogenen - 'mediatisierten' - Welt» vollziehen (Jörissen 2013). Medienbildung beinhaltet daher sowohl Bildungsprozesse, die über Medien stattfinden, als auch solche, die mit Medien stattfinden. Dies verdeutlicht, dass ein Ansatz der Medienbildung nicht nur instrumentell-qualifikatorische Aspekte in Betracht nimmt. Es geht vielmehr um die Transformation von Selbst- und Weltverhältnissen, also eine Transformation dessen, wie wir uns zur Welt und zu uns selbst in Beziehung setzen. Durch diese Perspektive wird deutlich, dass Menschen sich mit den Medien, die ihr Sein in der Welt beeinflussen, verändern (Marotzki und Jörissen 2009).

Dieses Verändern findet unter Bedingungen der technischen und gesellschaftlichen Beschleunigung von Alltags- und Arbeitszusammenhängen statt (Niesyto 2017), die zu medialer Aufmerksamkeitserregung oder einem Verlust von Reflexivität führen können (Rosa 2005; Niesyto 2012; Niesyto 2017). So sei der moderne Mensch, nach Sennett (1998), vor allem flexibel und immer nur auf den Moment, das Kurzfristige fokussiert. Bedingt vor allem durch den andauernden technologischen Fortschritt lässt sich besonders für die Kontexte der Medienpädagogik feststellen, dass die Anforderungsstrukturen einem ständigen Wandel und Fortschritt unterliegen. Dies stellt vor allem auch pädagogisch Tätige vor eine Herausforderung, bedingt durch das, was Rosa die Beschleunigung des sozialen Wandels nennt und was zu einer «Steigerung der Verfallsraten von handlungsorientierenden Erfahrungen und Erwartungen» (Rosa 2005, 133) führt. 
Diese Bedingungen verdeutlichen, dass im Kontext von Medienbildung ein materielles Bildungsverständnis unzureichend ist, da es in sich nicht reflexiv ist. Janetzko (2017) zeigt mit seiner Kritik der digitalen Kompetenzmodelle DigComp und MIL der EU und der UNESCO auf, dass diese Modelle häufig zu selbstbezogen und statisch seien. Während die Modelle Reflexivität von den Nutzerinnen und Nutzern erwarten, gilt dieses Argument auch in Bezug auf sie selbst. Reflexivität wird so als zentrales Merkmal im Umgang mit den sich immer fortwährend verändernden Technologien und Anforderungsprofilen im Rahmen dieser Technologien konstituiert.

Neben dem ständigen technologischen Fortschritt und den gesellschaftlichen Bedingungen der Beschleunigung stehen pädagogisch Tätige in einem weiteren Spannungsfeld. So zeigen Herzig und Aßmann (2012) die widersprüchlichen Anforderungen auf, die an Schule zwischen gesellschaftlichen und bildungspolitischen Erwartungen gerichtet werden. Diese Erwartungen werden von Lehrpersonen durchaus ambivalent aufgenommen. Während Erwartungen z. B. im Bereich des Cybermobbings nicht direkt in die relevanten Curricula oder Lehrpläne aufgenommen werden können, werden sie, ob implizit oder explizit artikuliert, von Lehrpersonen wahrgenommen und auch für notwendig erachtet (Herzig und Aßmann 2012). Gleichzeitig "sehen sie sich aufgrund allgemeiner beruflicher Anforderungen und z. B. (noch) fehlender Unterstützungs- und/oder Fortbildungsangebote nicht immer in der Lage, den Anforderungen in angemessener Weise nachzukommen» (Herzig und Aßmann 2012, 10). Medienpädagogische Elemente im Unterricht seien daher, auch begründet durch ihre Verankerung als Querschnittsaufgabe, häufig abhängig von der Sozialisation der Lehrpersonen. Die hier angebrachten Argumente haben deutlich gemacht, dass der Umgang mit Medien für pädagogisch Tätige einen wichtigen Bestandteil der Anforderungsstrukturen darstellt, die durch die gesellschaftlichen, bildungspolitischen und technologischen Veränderungen verstärkt werden. Zusammengefasst liesse sich dies so formulieren: Pädagogisch Tätige sollen mit Rücksicht auf den ständigen technologischen Fortschritt gesellschaftlichen und bildungspolitischen (z. B. Lehrpläne) Anforderungsprofilen folgend, reflektiert mit Medien umgehen und gleichzeitig einen reflektierten Umgang an die pädagogisch Anderen vermitteln können.

\section{Inklusion? Inklusion!}

Die Forderung nach Inklusiver Bildung stellt seit der Ratifikation der UN-Behindertenkonvention (UN-BRK) im Jahr 2009 eines der Megathemen dar, nachdem die Bundesrepublik und die Bundesländer formaljuristisch dazu verpflichtet sind, die Forderungen der UN-BRK progressiv umzusetzen. Dabei bezieht sich die UN-Konvention auf die Allgemeinen Menschenrechte und übersetzt diese für die Situation von Menschen mit Behinderungen (Degener 2009). Auch wenn die UN-BRK in Deutschland der Forderung nach Inklusiver Bildung qua menschenrechtlicher Legitimation 
Nachschub verliehen hat (Sturm 2016), besteht seit den 60er Jahren des 20. Jahrhunderts sowohl national als auch international eine Auseinandersetzung um die Forderung nach Inklusiver Bildung, die seit den 1990er Jahren Eingang in internationale Policies gefunden hat (z. B. Kiuppis 2014).

Ausgehend von dieser notwendigerweise normativ umschriebenen Anforderungssituation, die mit dem Begriff Inklusion einhergeht und momentan vor allem in Bezug auf das Schulsystem diskutiert wird, lassen sich verschiedene Spannungsfelder und Problematiken aufzeigen, die den Möglichkeitsraum für pädagogisches Handeln umreissen (Tenorth 2011; Katzenbach 2015).

So verweist ein systemtheoretischer Zugang zum Begriff der Inklusion darauf, dass dieser nicht ohne den Begriff der Exklusion denkbar ist (Stichweh 2007). Die Begriffe der In- und Exklusion fokussieren auf permanent und parallel stattfindende Prozesse des Ein- und Ausschlusses in Kommunikationszusammenhänge, die es innerhalb pädagogischer Praxis zu reflektieren gilt. Wenn der Anspruch der Inklusion immer auch mit Exklusion verbunden ist, so verweist die Rede von Inklusiver Bildung auf ein nicht zu erreichendes Ziel und verschleiert die Dynamik zwischen In- und Exklusion in pädagogischer Praxis.

Ganz ähnlich umschreiben Heinrich, Urban und Werning (2013) ein weites Verständnis von Inklusion, das als regulative Idee auf ein Minimum an Diskriminierung und ein Maximum an Teilhabe im Bildungssystem die entwickelten Modi der Handlungskoordination infrage stellt, was in einem engen Inklusionsverständnis insbesondere die Ausdifferenzierung von allgemeiner Schule und Sonderschule betrifft. Diese Modi haben sich, so die Autoren weiter, zur Bearbeitung des Spannungsfeldes zwischen dem pädagogischen Imperativ des Förderns der individuellen Entwicklung, der Aufgabe der Selektion und der Allokation des Schulsystems entwickelt. Ähnlich thematisiert z. B. Sturm (2015), dass Inklusion die «schulische 'Währung'» (Rabenstein et al. 2013, 674) der Leistungsorientierung infrage stellt. Auch Katzenbach (2015) weiter weist darauf hin, dass die Idee der egalitären Differenz, wie sie in der «Pädagogik der Vielfalt» von Prengel (2019) entworfen wird, dem meritokratischen Prinzip entgegensteht. Inklusion als menschenrechtliches Prinzip steht, so macht Katzenbach (2015) deutlich, der gesellschaftlichen Orientierung an Leistung entgegen. Zu beobachten ist allerdings, dass dieses Spannungsfeld zwischen Inklusion und Leistungsorientierung nicht auf gesellschaftlicher oder schulsystemischer Ebene bearbeitet wird. Stattdessen wird der Auftrag zur Umsetzung von Inklusion an Einzelschulen und Lehrkräfte weitergegeben, an die nun die Anforderung gestellt wird, mit diesem Widerspruch innerhalb schulischer Praxis umzugehen.

Dabei verweisen vor allem rekonstruktive Studien auf eine Differenz zwischen Norm und Praxis inklusiver Bildung (Asbrand et al. 2020). Eine Erklärung für diese Differenz kann im dialektischen Verhältnis zwischen der «globale[n] Durchsetzung transnationaler Programme und Modelle auf der einen und [der] überraschenden 
Beharrungskraft variierender sozial-kultureller Interrelations-Gefüge auf der anderen Seite» (Schriewer 2013, 35) gesehen werden. Die Anforderungen der UN-BRK müssen also lokal, auf Ebene einzelner Organisationen, in Interaktionen und Praktiken bearbeitet werden. Dabei reinterpretieren Akteurinnen und Akteure diese Anforderungen und passen diese in Praktiken und Logiken ein. So eindeutig die Anforderungen der UN-BRK zu sein scheinen, so vieldeutig werden diese durch verschiedene Akteursgruppen interpretiert: Der pädagogisch normative Diskurs versteht die UN-BRK als Legitimation der Forderungen der Inklusionspädagogik, die im Sinne eines weiten Inklusionsverständnisses die Entwicklung einer Schule für Alle fordert. Im Gegensatz dazu wird die UN-BRK im Kontext der Schuladministration auf die Differenzkategorie Beeinträchtigung oder Behinderung bezogen und als Forderung nach der Inklusiven Beschulung dieser Schülerinnen- und Schülergruppe in der allgemeinen Schule reinterpretiert (Gasterstädt 2019). Die beschriebene Differenz zwischen Norm und Praxis lässt sich dementsprechend als doppelte Differenz zwischen der strukturell eingebetteten Praxis, der Norm der UN-BRK und des pädagogisch-normativen Diskurses sowie der schulsystemisch gebrochenen Norm der Inklusiven Beschulung fassen. Letztere bestimmt allerdings den Möglichkeitsrahmen pädagogischen Handelns, da an diese schulsystemspezifische Reinterpretation von Inklusiver Bildung Strukturvorgaben und Steuerungsimpulse innerhalb der Bundesländer ansetzen. Hier lässt sich dann auch die Frage nach dem Mandat pädagogischen Handelns im Kontext Inklusiver Bildung anschliessen.

\section{Inklusiv-mediale Bildung?}

Denkt man diese Anforderungsstrukturen im Sinne einer inklusiv-medialen Bildung nun zusammen, werden sechs Aspekte deutlich. So wurde weiter oben aufgezeigt, dass Bildungsprozesse sowohl mit Medien als auch in/über Medien stattfinden. Mit dieser doppelten Bestimmung von Medienbildung lassen sich zwei erste Schlüsse auf die Anforderungsstruktur inklusiv-medialer Bildung ziehen.

Erstens ist davon auszugehen, dass der Einsatz von Medien(-technik) Bildungsprozesse ermöglichen, aber auch verhindern kann. Neben der Diskussion um die Gestaltung barrierefreier Informationstechnologien und die Möglichkeiten der technischen Unterstützung von Kommunikation soll dabei folgender Aspekt betont werden: So kann der Einsatz verschiedener Medien in Lernsettings die Möglichkeiten der Teilhabe an Bildungsinhalten erweitern. Beispiele aus Schottland zeigen, dass die Teilhabe an Bildungsinhalten trotz eingeschränkten Schriftspracherwerbs im Rahmen der Sekundarstufe über gängige technische Hilfsmittel (z. B. Sprachausgabe mittels Tablets) ermöglicht werden kann (Nisbet 2016). Komplementär dazu kann die Fokussierung auf den Einsatz von Medien, die entsprechende Lesekompetenzen voraussetzen, die Teilhabe an diesen Inhalten erschweren oder verhindern. 
Zweitens verweist Jörissen (2013) darauf, dass Bildung auch in/durch Medien stattfindet. So findet Bildung vermittelt in Medien statt und es gilt als Aufgabe insbesondere von Bildungsinstitutionen, einen kompetenten Umgang medialer Inhalte zu vermitteln. Mit Bezug auf inklusionspädagogische Konzeptionen hiesse das auch, insbesondere Fragen der Konstruktion von Differenzen und Ungleichheiten in medialen Darstellungen und die Verwendung oder Thematisierung stereotyper Vorstellung und daran anschliessender Vorurteile zu reflektieren (Trescher und Börner 2016). Angesichts der Wirkmächtigkeit (medialer) Diskurse, die sich entsprechender Stereotype und Vorurteile bedienen, stellt es sich allerdings als fraglich dar, inwiefern die Anforderung zur Vermittlung eines reflektierten Umgangs mit solchen medial vermittelten Inhalten in Lernsettings von z. B. Lehrkräften, die selbst der Wirkmächtigkeit dieser Diskurse ausgesetzt sind, erfüllt werden kann.

Drittens lässt sich sowohl im Rahmen inklusiver, als auch im Rahmen von medialer Bildung das folgende Phänomen beobachten: Die Pluralität des Diskurses trifft auf eine pädagogische Praxis, deren Bezugsmöglichkeiten dadurch mindestens ebenso plural sind. Im Zuge von medialer Bildung lässt sich zum Beispiel am Diskurs zum Begriff der Medienkompetenz verdeutlichen (Moser 2010), dass pädagogisch Tätige schliesslich vor die Herausforderung gestellt werden, medienkompetent zu sein, ohne dass Medienkompetenz eindeutig definiert wäre. Im Inklusionsdiskurs lässt sich diese Schablone genauso beschreiben, in Anbetracht der diversen (wissenschaftlichen) Perspektiven darüber, was Inklusion überhaupt ist (Hinz 2013), werden pädagogisch Tätige nicht nur vor die Herausforderung gestellt, inklusiv zu agieren, sondern auch hier erst einmal zu definieren, was Inklusion überhaupt bedeutet.

Viertens stehen die beiden Felder auch in einer Art Konkurrenzverhältnis zueinander. Dies zeigt sich im pädagogischen Alltag bereits im Bereich der Ressourcenausstattung: Räume in denen auf angemessener Ebene sowohl Inklusion als auch Medienbildung verhandelt werden können und die beiden Themen daher wirklich voneinander profitieren können, gibt es nicht genug. Wie schon zuvor gezeigt, gibt es sehr wohl einen Mehrwert in der Verbindung der beiden Themen, jedoch muss dies auch über eine rein instrumentell-qualifikatorische Perspektive von Mediennutzung für inklusive Ziele hinausgehen. An dieser Stelle werden schliesslich auch Ressourcenfragen (etwa Zeit, Geld, Infrastrukturen, Expertisen) zentral und verdeutlichen noch einmal das Konkurrenzverhältnis, das im Hinblick auf die Umsetzung im pädagogischen Alltag zwischen den beiden viel verhandelten Themen entsteht.

Fünftens stellt sich die Frage, worin sich die Schwierigkeiten bei der Realisierung dieser Anforderungsstrukturen abseits der konkreten Ressourcenausstattung gründen. Hierbei erweist es sich als instruktiv, auf die Ursprünge dieser zusätzlichen Anforderungsstrukturen einzugehen. Sie basieren auf der strukturellen Kopplung des Bildungssystems mit anderen gesellschaftlichen Teilbereichen. Es lässt sich festhalten, dass schon bei der internen Verarbeitung der Megathemen Aushandlungsprozesse 
auftreten, die zur Priorisierung einzelner Facetten des Diskurses innerhalb eines Funktionssystems führen können. Diese auf spezifischen Eigenlogiken basierenden Akzentuierungen haben Folgen für die Kommunikation zwischen den Funktionssystemen. Je nach Schwerpunktsetzung innerhalb der Teilsysteme werden die Pluralität des Diskurses und die damit verbundenen Bezugsmöglichkeiten im jeweils anderen Teilsystem vorstrukturiert. Im Bereich der Digitalisierung entstammen wesentliche Impulse für das Bildungssystem (bspw. arbeitsmarktfähige Medienkompentenzmodelle oder Softwareeinsatz zur Steigerung der individuellen Produktivität) dem Wirtschaftssystem. Hingegen erweisen sich in Bezug auf Inklusion die Akzentuierungen des Rechtssystems als wirkungsvoll, da erst mit der Ratifizierung der UN-BRK das Thema Inklusion bildungspolitische Relevanz erhalten hat. Genau diese Konstellation der Präformierung von Bezugspotentialen führt durch die strukturelle Kopplung der Funktionssysteme zu Irritationen, da unterschiedliche und potentiell divergierende Eigenlogiken aufeinanderprallen.

Sechstens werden die Irritationen durch einen im vorigen Abschnitt zunächst vereinfacht dargestellten Umstand potenziert: Die erwähnten Aushandlungsprozesse zur internen Verarbeitung der Megathemen innerhalb eines Funktionssystems führen nicht zu einem zeitlich konstanten Ergebnis. Die Unschärfe in der Begriffsbestimmung von Inklusion und Digitalisierung zeigt sich somit nicht nur als konstitutiv für das Bildungssystem, sondern auch für die mit ihm verbundenen Teilsysteme. Darin wird deutlich, dass auch die Erwartungen an das Bildungssystem bei der Umsetzung inklusiv-medialer Bildung aufgrund der schlichten Tatsache variieren, dass selbst in den verschiedenen Funktionssystemen die Pluralität des jeweiligen Diskurses zu Irritationen führt.

\section{6. $\quad$ Fazit}

Auf der Basis von systemtheoretischen Überlegungen wurden strukturelle gesellschaftliche Transformationen verdeutlicht und professionstheoretisch mit Fokus auf die Konturen der Anforderungsstrukturen gerahmt. Hierbei liess sich feststellen, dass die Veränderungen der Anforderungsstrukturen für pädagogisch Tätige in ein Spannungsfeld eingelagert sind, das in den Wechselbeziehungen aus pädagogischer Praxis, (Bildungs-)Politik und (erziehungs-)wissenschaftlichem Diskurs verhandelt wird. Anschliessend wurden in Bezug zu dieser Triade die relevanten Veränderungen und Herausforderungen für die Anforderungsstruktur pädagogisch Tätiger für den Rahmen des Digitalisierungsdiskurses sowie des Inklusionsdiskurses skizziert. Dabei wurde deutlich, inwiefern die Anforderungsstrukturen sowohl im Bereich von Inklusion als auch im Bereich medialer Bildung im Zuge von Digitalisierung komplexen Transformationen ausgesetzt sind. Es wurde aufgezeigt, dass nicht nur die 
Auseinandersetzung mit den beiden Themen als Herausforderung an pädagogisches Handeln begriffen werden kann, sondern dass sich diese Anforderungen durch das parallele Auftreten beider Themen potenzieren.

Als Antwort auf diese Anforderungen wird im (erziehungs-)wissenschaftlichen Diskurs um die Aus-, Weiter- und Fortbildung pädagogischer Fachkräfte insbesondere Reflexion als zentrale Aufgabe und Kompetenz diskutiert (Bolten und Rott 2018; Schuppener 2014). In Anbetracht der skizzierten strukturell bedingten Spannungsfelder inklusiv-medialer Bildung sehen wir allerdings die Gefahr, mit der Forderung nach Reflexion als pädagogischer Kompetenz die strukturellen Herausforderungen zu verdecken, die mit diesen verbunden sind und nicht auf Ebene pädagogischen Handelns bearbeitet werden können.

Des Weiteren wird mit den geschilderten Anforderungssituationen im Hinblick auf inklusive Bildung sowie Digitalisierung deutlich, dass sich diese sowohl an die pädagogische Praxis, die (Bildungs-)Politik als auch die (Erziehungs-)Wissenschaft richten. Insbesondere an das Wissenschaftssystem als Ebene zweiter Beobachtung, auf die sich professionelle pädagogische Praxis beziehen soll, stellt sich in Anbetracht der Pluralität und Situativität von Wissensproduktion in der sogenannten Postmoderne einmal mehr die Anforderung, selbstkritisch mit den strukturellen Bedingungen, in denen Wissen produziert wird, umzugehen.

\section{Literatur}

Asbrand, Barbara, Julia Gasterstädt, Anja Hackbarth, und Matthias Martens. 2020. «Was bewegt Inklusion? Inklusiver Unterricht aus schul- und sonderpädagogischer Perspektive». In Bewegungen. Beiträge zum 26. Kongress der Deutschen Gesellschaft für Erziehungswissenschaft, herausgegeben von Nicolle Pfaff et al. 514-529. Opladen: Barbara Budrich.

Bauman, Zygmunt. 2009. Gemeinschaften. Frankfurt am Main: Suhrkamp.

Bettinger, Patrick. 2018. Praxeologische Medienbildung. Theoretische und empirische Perspektiven auf sozio-mediale Habitustransformationen. Wiesbaden: Springer VS. https://doi. org/10.1007/978-3-658-21849-2.

BMBF. 2019. Wissenswertes zum DigitalPakt Schule». https://www.bmbf.de/de/wissenswerteszum-digitalpakt-schule-6496.php.

Bolten, Ricarda, und Karin Julia Rott. 2018. «Medienpädagogische Kompetenz: Anforderungen an Lehrende in der Erwachsenenbildung - Perspektiven der Praxis». MedienPädagogik 30: 137-153. https://doi.org/10.21240/mpaed/30/2018.03.05.X.

Castells, Manuel. 2017. Der Aufstieg der Netzwerkgesellschaft. Das Informationszeitalter. Wirtschaft, Gesellschaft, Kultur. 2. Auflage. Wiesbaden: Springer VS. https://doi. org/10.1007/978-3-658-11322-3. 
Damberger, Thomas. 2018. «Mediendidaktik im Digitalzeitalter». In Spannung? Potenziale! Spannungsfelder und Bildungspotenziale des Digitalen, herausgegeben von Thomas Knaus und Olga Engel, 77-100. München: Kopaed.

Dander, Valentin. 2020. «Sechs Thesen zum Verhältnis von Digitalisierung, Digitalisierung und Bildung». In Digitalisierung - Subjekt - Bildung, herausgegeben von Valentin Dander, Patrick Bettinger, Estella Hebert, Christian Leineweber und Klaus Rummler. Opladen: Barbara Budrich. https://doi.org/10.2307/j.ctvvb7n3h.5.

Degener, Theresia. 2009. «Die UN-Behindertenrechtskonvention als Inklusionsmotor». Recht der Jugend und des Bildungswesens 57 (2): 200-219.

Gasterstädt, Julia. 2019. Der Komplexität begegnen und Inklusion steuern. Eine Situationsanalyse zur Umsetzung von Artikel 24 der UN-BRK in zwei Bundesländern in Deutschland. Wiesbaden: Springer VS. https://doi.org/10.1007/978-3-658-27838-0.

Geissler, Birgit. 2013. "Professionalisierung und Profession. Zum Wandel klientenbezogener Berufe im Übergang zur post-industriellen Gesellschaft». die hochschule 2: 19-32.

Heinrich, Martin, Michael Urban, und Rolf Werning. 2013. «Grundlagen, Handlungsstrategien und Forschungsperspektiven für die Ausbildung und Professionalisierung von Fachkräften für inklusive Schule». In Inklusive Bildung professionell gestalten, herausgegeben von Hans Döbert und Horst Weishaupt, 69-133. Münster u.a.: Waxmann.

Herzig, Bardo, und Sandra Aßman. 2012. «Medienpädagogik und Schule». In EEO Enzyklopädie Erziehungswissenschaft Online. Weinheim: Beltz Juventa.

Hinz, Andreas. 2013. «Inklusion - von der Unkenntnis zur Unkenntlichkeit!? Kritische Anmerkungen zu einem Jahrzehnt Diskurs über schulische Inklusion in Deutschland». Zeitschrift für Inklusion 1. https://www.inklusion-online.net/index.php/inklusion-online/article/ view/26/26.

Hof, Christiane. 2009. Lebenslanges Lernen. Eine Einführung. Stuttgart: Kohlhammer.

Hughes, Everett Cherrington. 1993. The sociological eye. Selected papers. New Brunswick: Transaction Publishers.

Janetzko, Dietmar. 2017. "Social Bots and Fake News as (not) seen from the Viewpoint of Digital Education Frameworks». MedienPädagogik: 61-80. https://doi.org/10.21240/ mpaed/00/2017.07.05.X.

Jörissen, Benjamin. 2013. «'Medienbildung‘ in 5 Sätzen». https://joerissen.name/medienbildung/medienbildung-in-5-satzen/.

Katzenbach, Dieter. 2015. «Zu den Theoriefundamenten der Inklusion. Eine Einladung zum Diskurs aus der Perspektive der kritischen Theorie». In Herausforderung Inklusion. Theoriebildung und Praxis, herausgegeben von Irmtraud Schnell, 19-33. Bad Heilbrunn: Klinkhardt.

Kiuppis, Florian. 2014. Heterogene Inklusivität, inklusive Heterogenität. Bedeutungswandel imaginierter pädagogischer Konzepte im Kontext Internationaler Organisationen. Münster: Waxmann.

Luhmann, Niklas. 1997. Die Gesellschaft der Gesellschaft. Frankfurt am Main: Suhrkamp. 
Marotzki, Winfried, und Benjamin Jörissen. 2008. «Wissen, Artikulation und Biographie: theoretische Aspekte einer Strukturalen Medienbildung». In Pädagogische Medientheorie, herausgegeben von Johannes Fromme und Werner Sesink, 51-70. Wiesbaden: VS Verlag. https://doi.org/10.1007/978-3-531-90971-4.

Marotzki, Winfried und Benjamin Jörissen. 2009. Medienbildung - Eine Einführung. Bad Heilbrunn: Klinkhardt UTB.

Moser, Heinz. 2010. Einführung in die Medienpädagogik. Wiesbaden: VS Verlag. https://doi. org/10.1007/978-3-531-92215-7.

Niesyto, Horst. 2012. "Bildungsprozesse unter den Bedingungen medialer Beschleunigung». In Raum, Zeit, Medienbildung. Untersuchungen zu medialen Veränderungen unseres Verhältnisses zu Raum und Zeit. Reihe Medienbildung und Gesellschaft. Bd. 23, herausgegeben von Bukow, Gerhard, Johannes Fromme und Benjamin Jörissen, 47-66. Wiesbaden: VS Verlag. https://doi.org/10.1007/978-3-531-19065-5.

Niesyto, Horst. 2017. «Medienpädagogik und digitaler Kapitalismus. Für die Stärkung einer gesellschafts- und medienkritischen Perspektive». MedienPädagogik: Zeitschrift für Theorie und Praxis der Medienbildung 27 (Spannungsfelder \& blinde Flecken): 1-29. https://doi. org/10.21240/mpaed/27/2017.01.13.X.

Nisbet, Paul. 2016. «Raising attainment through Assistive Technology». https://www.callscotland.org.uk/blog/raising-attainment-through-assistive-technology/.

Nittel, Dieter. 2011. «Von der Profession zur sozialen Welt pädagogisch Tätiger? Vorarbeiten zu einer komparativ angelegten Empirie pädagogischer Arbeit». Zeitschrift für Pädagogik 57: 40-59.

Nittel, Dieter, und Julia Schütz. 2013. «Zwischen Verberuflichung und Professionalität. Professionalisierungsdynamiken in der sozialen Welt der Erzieherinnen und Weiterbildner». In Engagement für die Erwachsenenbildung. Ethische Bezugnahmen und demokratische Verantwortung, herausgegeben von Bernd Käpplinger, Steffi Robak und Sabine Schmidt-Lauff, 111-129. Wiesbaden: Springer VS. https://doi.org/10.1007/978-3-531-19116-4.

Nittel, Dieter, und Astrid Seltrecht. 2008. «Der Pfad der „individuellen Professionalisierung“: ein Beitrag zur kritisch-konstruktiven erziehungswissenschaftlichen Berufsgruppenforschung». BIOS - Zeitschrift für Biographieforschung, Oral History und Lebensverlaufsanalysen 21: 124-145.

Prengel, Annedore. 2019. Pädagogik der Vielfalt. Verschiedenheit und Gleichberechtigung in Interkultureller, Feministischer und Integrativer Pädagogik. 4. Auflage. Wiesbaden: VS Verlag für Sozialwissenschaften. https://doi.org/10.1007/978-3-658-21947-5.

Rabenstein, Kerstin, Sabine Reh, Norbert Ricken, und Till-Sebastian Idel. 2013. «Ethnographie pädagogischer Differenzordnungen. Methodologische Probleme einer ethnographischen Erforschung der sozial selektiven Herstellung von Schulerfolg im Unterricht». Zeitschrift für Pädagogik 59 (5): 668-690.

Rosa, Hartmut. 2005. Beschleunigung. Die Veränderung der Zeitstrukturen in der Moderne. Frankfurt am Main: Suhrkamp. 
Schriewer, Jürgen. 2013. «Vergleichende Erziehungswissenschaft als Forschungsfeld». In Kulturvergleich in der qualitativen Forschung: Erziehungswissenschaftliche Perspektiven und Analysen, herausgegeben von Merle Hummrich und Sabine Rademacher, 15-41. Wiesbaden: Springer VS. https://doi.org/10.1007/978-3-531-18937-6.

Schuppener, Saskia. 2014. «Inklusive Schule: Anforderungen an Lehrer_innenbildung und Professionalisierung». Zeitschrift Für Inklusion (1-2). https://www.inklusion-online.net/index. php/inklusion-online/article/view/220.

Sennett, Richard. 1998. Der flexible Mensch. Die Kultur des neuen Kapitalismus. Berlin: Berlin Verlag.

Stichweh, Rudolf. 1996. «Professionen in einer funktional differenzierten Gesellschaft». In Pädagogische Professionalität, herausgegeben von Arno Combe und Werner Helsper, 41-69. Frankfurt am Main: Suhrkamp.

Stichweh, Rudolf. 2007. Inklusion und Exklusion in der Weltgesellschaft - am Beispiel der Schule und des Erziehungssystems. Wiesbaden: Springer VS. https://doi.org/10.1007/978-3-53190627-0_5.

Strauss, Anselm L. 1993. Continual Permutations of Action. New York: de Gruyter.

Sturm, Tanja. 2015. Inklusion: «Kritik und Herausforderung des schulischen Leistungsprinzips». Erziehungswissenschaft 26 (51): 25-32.

Sturm, Tanja. 2016. «Phasen der Entwicklung inklusiver Bildung». In Handbuch Inklusion und Sonderpädagogik, herausgegeben von Ingeborg Hedderich, Gottfried Biewer, Judith Hollenweger und Reinhard Markowetz, 179-183. Bad Heilbrunn: Klinkhardt.

Tenorth, Heinz-Elmar. 2011. «Inklusion im Spannungsfeld von Universalisierung und Individualisierung-Bemerkungen zu einem pädagogischen Dilemma». http://www.schulentwicklung.bayern.de/unterfranken/userfiles/SETag2011/Tenorth-Inklusion-Wuerzburg-2011. pdf.

Tippelt, Rudolf. 2018. «Professionsforschung und Bildung». In Handbuch Bildungsforschung, 4. Aufl., herausgegeben von Rudolf Tippelt und Bernhard Schmidt-Hertha, 649-666. Wiesbaden: VS. https://doi.org/10.1007/978-3-531-19981-8.

Trescher, Hendrik, und Michael Börner. 2016. «Repräsentanz und Subjektivität im Kontext geistiger Behinderung». Zeitschrift Für Inklusion 1. https://www.inklusion-online.net/index. php/inklusion-online/article/view/341.

Wahl, Johannes. 2017. Lebenslanges Lernen zwischen Bildungspolitik und pädagogischer Praxis. Die Verankerung in pädagogischen Arbeitsfeldern. Bielefeld: Bertelsmann. https://doi. org/10.3278/6004502w. 